\title{
Incidence of Fractures after Cardiac and Lung Transplantation: A Single Center Experience
}

\author{
Aileen Hariman, ${ }^{1}$ Charles Alex, ${ }^{2}$ Alain Heroux, ${ }^{3}$ and Pauline Camacho ${ }^{1}$ \\ ${ }^{1}$ Department of Endocrinology, Loyola University Medical Center, Maywood, IL 60153, USA \\ ${ }^{2}$ Department of Pulmonology/Critical Care, Advocate Christ Medical Center, Oak Lawn, IL 60453, USA \\ ${ }^{3}$ Department of Cardiology, Loyola University Medical Center, Maywood, IL 60153, USA \\ Correspondence should be addressed to Aileen Hariman; ahariman@lumc.edu
}

Received 20 November 2013; Revised 17 March 2014; Accepted 19 March 2014; Published 22 April 2014

Academic Editor: Jun Iwamoto

Copyright (C) 2014 Aileen Hariman et al. This is an open access article distributed under the Creative Commons Attribution License, which permits unrestricted use, distribution, and reproduction in any medium, provided the original work is properly cited.

\begin{abstract}
Osteoporotic fractures are well-known complications of organ transplantation. Fracture rates up to $35 \%$ have been previously reported following heart and lung transplantations. Our institutional pretransplant protocols include DXA scans, vitamin D screening, and appropriate antiresorptive therapy. We aimed to assess the incidence of fragility fractures following cardiac or lung transplantation. In a retrospective study 210 electronic medical records of patients who underwent LT (110 men, 100 women) and 105 HT (88 men, 17 women) between 2005 and 2010 were analyzed. Both clinical and radiographic fractures were recorded. DXA scans were obtained immediately after transplant. 17 out of 210 LT patients (8.0\%) had fractures after transplantation and 9 out of 105 HT patients $(8.6 \%)$ had fractures. The median time to the first fracture was 12 months and the mean time was 18 months for both LT and HT. In the HT recipients, the median femoral neck T score was statistically lower in the fracture group versus the nonfracture group. Similar results were seen in the LT patients. Conclusion. Our findings demonstrate a much lower incidence of fractures in heart and lung transplant recipients in comparison with earlier reports. Comprehensive bone care and early initiation of antiresorptive therapy are possible contributors to these improved outcomes.
\end{abstract}

\section{Introduction}

Osteoporosis and the development of fractures are wellknown complications in patients following organ transplantation and affect their quality of life. In addition to risk factors such as Caucasian race, female gender, estrogen and androgen deficiency, immobilization, vitamin D deficiency, and low bone mineral density prior to organ transplantation, patients who undergo transplantation are placed on immunosuppressive therapy which may contribute to the pathogenesis and rapid development of fractures.

Patients awaiting cardiac transplantation are at risk for osteoporosis as patients with end-stage congestive heart failure have been exposed to loop diuretics resulting in a negative calcium balance. In addition, cardiac cachexia, reduced exercise levels, smoking, alcohol abuse, hypogonadism related to debilitating illness, and anticoagulation may contribute to bone loss. Low levels of 25-hydroxyvitamin D and 1,25dihydroxyvitamin $\mathrm{D}$ are associated with secondary hyperparathyroidism and are common in these patients $[1,2]$.

Patients who undergo lung transplantation are already at increased risk for osteoporosis due to malnutrition, malabsorption from cystic fibrosis, history of smoking, hypogonadism, hypovitaminosis $\mathrm{D}$, and exposure to glucocorticoids [3].

At Loyola University Medical Center, pretransplant protocols routinely include DXA scans and aggressive vitamin D screening prior to and immediately after transplantation. Antiresorptive medications are widely utilized. The aim of this study was to assess the incidence of fragility fractures following cardiac or lung transplantation. 


\section{Materials and Methods}

2.1. Study Patients. The study population included all adults who underwent single or bilateral lung transplantation or underwent heart transplantation at Loyola University Medical Center between 2005 and 2010. All fragility fractures were included regardless of cause. Patients less than 18 years of age were excluded from the analysis.

Patients who underwent lung transplantation were grouped under different classifications by diagnosis, such as chronic obstructive pulmonary disease, interstitial fibrosis, and others. Similarly, patients who underwent heart transplantation were grouped under different classifications such as ischemic cardiomyopathy, dilated cardiomyopathy, and others.

2.2. Study Design. This was a retrospective observational study conducted between the years 2005-2010. Information was obtained and analyzed through electronic medical records. Both clinical and radiographic fractures through review of medical records were recorded.

The occurrence of fractures was assessed through chart review in the EPIC database, by reviewing progress notes, and radiological evidence of fracture. X-rays, MRI, CT, and DXA scans were reviewed.

Baseline data was obtained from all patients, including age at surgery, sex, BMI, prior smoking history and tobacco exposure, current use of glucocorticoids, menopausal status at the time of transplant (if applicable), and prior history of fracture.

Immunosuppression therapy for heart transplantation patients included mycophenolate, prednisone, and either cyclosporine, tacrolimus, or sirolimus. Immunosuppression therapy for lung transplantation patients included tacrolimus, prednisone, and azathioprine or mycophenolate.

2.3. Osteoporosis Assessment and Treatment. Patients were reviewed to assess whether they have had a DXA scan immediately following transplantation. Patients were assessed on whether they are currently using calcium and vitamin D supplementation and/or antiresorptive medication use based on objective evidence of osteopenia or osteoporosis.

2.4. Laboratory Data. Patients were reviewed to assess baseline and posttransplantation serum calcium, phosphorus, bone-specific alkaline phosphatase, albumin, creatinine, PTH levels, and 25-hydroxyvitamin D.

2.5. Data Analysis. Descriptive statistics such as mean and SD (standard deviation) or median and IQR (interquartile range) were used to summarize patient characteristics for continuous variables, whereas frequencies and percentages were used for categorical variables. Due to the small sample sizes, Wilcoxon rank sum tests were generally used to assess differences between fractured and nonfractured subjects, with respect to continuous variables (i.e., age at transplant). Chi-square or Fisher's exact tests were used to assess the association between categorical variables (i.e., gender) and the occurrence of fracture. Data was stored in excel format, and the statistical analysis was carried out using statistical analysis software (SAS).

\section{Results}

Baseline demographics, clinical characteristics, reasons for transplantation, and results prior to and following lung transplantation are listed in Tables 1 and 2. Similar data in those who underwent heart transplantation are listed in Tables 3 and 4.

3.1. Lung Transplantation. Out of 210 lung transplant recipients, 17 patients $(8.0 \%)$ had fractures after transplantation. The incidence of fracture in lung transplant patients in years 1 to 5 was $4.8 \%, 1.0 \%, 0.0 \%, 1.0 \%$, and $1.9 \%$, respectively. The median time to the first fracture was 12 months and the mean time was $17.88 \pm 15.19$ months. Out of the 210 lung transplant recipients, 100 patients were female, and $8 \%$ of female patients incurred fractures. Out of the 110 male lung transplant recipients, $8.2 \%$ of male recipients incurred fractures.

The median pretransplant lumbar spine (LS) T score did not differ in those who fractured versus those who did not $(-1.60[-2.40-0.10]$ versus $-1.00[-2.00-0.10], P=.29)$. The median pretransplant femoral neck $\mathrm{T}$ score did not differ in those who fractured versus those who did not $(-2.40[-2.70-$ $-1.80]$ versus $-1.50[-2.10--0.60], P=.053)$.

The median posttransplant LS T score did not differ in those who fractured versus those who did not $(-1.50$ $[-2.40--0.25]$ versus $-1.00[-2.00-0.00], P=.29)$. The median femoral neck $\mathrm{T}$ score was statistically lower in the fracture group versus the nonfracture group $(-2.75[-3.32-$ $-2.0]$ versus $-1.60[-2.43--1.00], P=.01)$.

The median age (in years) of lung transplant recipients who fractured was not significantly different than those who did not (58 [50-59] versus 56 [49-62], $P=.96)$. The use of bisphosphonates in fracture and nonfracture groups did not differ in lung transplant patients $(53.0 \%$ versus $50 \%$, $P=.78)$. Calcium and vitamin $\mathrm{D}$ use did not differ between fracture and nonfracture groups (94\% versus 90\%, $P=$ .99). Glucocorticoid use did not differ between fracture and nonfracture groups (100\% versus $91 \%, P=.37$ ).

Fracture locations varied, including 4 hip (23.5\%), 3 vertebral (17.6\%), 3 rib (17.6\%), 2 upper extremity (11.76\%), 2 wrist (11.76\%), 2 lower extremity (11.76\%), and 1 foot $(5.89 \%)$ fracture. None of the patients incurred multiple fractures that took place concurrently. None of these patients incurred subsequent fractures. Etiology of those who fractured after transplant included 8 patients with chronic obstructive pulmonary disease (COPD), 5 with idiopathic pulmonary fibrosis (IPF), 2 with sarcoidosis, 1 with scleroderma, and 1 with alpha 1 antitrypsin deficiency (A1AD). Out of the 11 patients who had fractures prior to lung transplantation, none had fractures following transplantation.

3.2. Heart Transplantation. Out of 105 heart transplant patients, 9 patients $(8.6 \%)$ had fractures. The incidence of 
TABLE 1: Comparison of those with fractures versus no fractures in the lung transplant population.

\begin{tabular}{|c|c|c|c|c|}
\hline & Fracture $n=17$ & Nonfracture $n=193$ & Test statistic $(\mathrm{df})$ & $P$ \\
\hline \multicolumn{5}{|l|}{ Demographics } \\
\hline Mdn age (years) (IQR) & $\begin{array}{c}58(50-59) \\
\quad n=17\end{array}$ & $\begin{array}{c}56(49-62) \\
n=192\end{array}$ & $1772.5(209)^{1}$ & .96 \\
\hline Mdn BMI (IQR) & $\begin{array}{c}23.65(21.51-25.35) \\
n=16\end{array}$ & $\begin{array}{c}25.01(20.97-27.35) \\
n=187\end{array}$ & $1381.00(203)^{1}$ & .27 \\
\hline Females & $8 / 17(47 \%)$ & $92 / 192(48 \%)$ & $0.005(1)^{2}$ & .95 \\
\hline Menopause & $1 / 8(13 \%)$ & $32 / 91(35 \%)$ & $1.70(1)^{3}$ & .26 \\
\hline Ex-smokers & $13 / 17(77 \%)$ & $127 / 192(66 \%)$ & $0.75(1)^{2}$ & .39 \\
\hline Pretransplant fracture & $0 / 16(0 \%)$ & $11 / 192(6 \%)$ & $0.97(1)^{3}$ & .99 \\
\hline \multicolumn{5}{|l|}{ Reason for transplantation } \\
\hline Chronic obstructive pulmonary disease & $8 / 17(47 \%)$ & 60/193 (31\%) & & \\
\hline Idiopathic pulmonary fibrosis & $5 / 17(29 \%)$ & $64 / 193(33 \%)$ & $2.04(2)^{2}$ & .36 \\
\hline Other & $4 / 17(24 \%)$ & $69 / 193(36 \%)$ & & \\
\hline \multicolumn{5}{|l|}{ Pretransplant values } \\
\hline Mdn LS T score (IQR) & $\begin{array}{c}-1.60(-2.40-0.10) \\
n=13\end{array}$ & $\begin{array}{c}-1.00(-2.00-0.10) \\
n=131\end{array}$ & $791.50(144)^{1}$ & .29 \\
\hline Mdn femoral neck T score (IQR) & $\begin{array}{c}-2.40(-2.70--1.80) \\
n=13\end{array}$ & $\begin{array}{c}-1.50(-2.10--0.60) \\
n=134\end{array}$ & $678.00(147)^{1}$ & .053 \\
\hline Mdn serum calcium (mg/dL) (IQR) & $\begin{array}{c}8.30(7.80-8.80) \\
n=17\end{array}$ & $\begin{array}{c}8.20(7.80-9.00) \\
n=191\end{array}$ & $1740.50(208)^{1}$ & .88 \\
\hline Mdn serum phosphorus (mg/dL) (IQR) & $\begin{array}{c}3.15(2.3-3.9) \\
n=8\end{array}$ & $\begin{array}{c}3.60(3.10-4.20) \\
n=93\end{array}$ & $292.00(101)^{1}$ & .15 \\
\hline Mean serum albumin (gm/dL) (SD) & $\begin{array}{c}2.80(0.45) \\
n=14\end{array}$ & $\begin{array}{c}2.62(0.60) \\
n=138\end{array}$ & $-1.11(150)^{4}$ & .27 \\
\hline Mdn serum bone-specific alk. phosphatase (mcg/L) (IQR) & - & $\begin{array}{c}10.10(9.40-13.10) \\
n=13\end{array}$ & - & - \\
\hline Mdn serum parathyroid hormone (pg/mL) (IQR) & $\begin{array}{c}50.00(41.00-53.00) \\
n=5\end{array}$ & $\begin{array}{c}41.00(30.00-65.00) \\
n=87\end{array}$ & $277.00(92)^{1}$ & .45 \\
\hline Mdn serum vitamin D 25-OH (ng/mL) (IQR) & $\begin{array}{c}29.00(20.00-38.00) \\
n=2\end{array}$ & $\begin{array}{c}28.50(18.50-37.00) \\
n=56\end{array}$ & $64.00(58)^{1}$ & .85 \\
\hline Mdn serum creatinine (mg/dL) (IQR) & $\begin{array}{c}0.73(0.60-0.94) \\
n=17\end{array}$ & $\begin{array}{c}0.81(0.70-1.00) \\
n=192\end{array}$ & $1511.00(209)^{1}$ & .25 \\
\hline \multicolumn{5}{|l|}{ Posttransplant medications } \\
\hline Bisphosphonate & $9 / 17(53 \%)$ & $95 / 192(50 \%)$ & $0.08(1)^{2}$ & .78 \\
\hline Calcium and vitamin $\mathrm{D}$ & $16 / 17(94 \%)$ & $165 / 183(90 \%)$ & $0.28(1)^{3}$ & .99 \\
\hline Glucocorticoid & $17 / 17(100 \%)$ & $175 / 193(91 \%)$ & $1.73(1)^{3}$ & .37 \\
\hline \multicolumn{5}{|l|}{ Posttransplant values } \\
\hline Mdn LS T score (IQR) & $\begin{array}{c}-1.50(-2.40--0.25) \\
n=12\end{array}$ & $\begin{array}{c}-1.00(-2.00-0.00) \\
n=98\end{array}$ & $556.00(110)^{1}$ & .29 \\
\hline Mdn femoral neck T score (IQR) & $\begin{array}{c}-2.75(-3.32--2.0) \\
n=12\end{array}$ & $\begin{array}{c}-1.60(-2.43--1.00) \\
n=98\end{array}$ & $376.00(110)^{1}$ & .01 \\
\hline Mdn serum calcium (mg/dL) (IQR) & $\begin{array}{c}8.90(8.50-9.10) \\
n=17\end{array}$ & $\begin{array}{c}8.80(8.50-9.20) \\
n=188\end{array}$ & $1713.00(205)^{1}$ & .87 \\
\hline Mdn serum phosphorus (mg/dL) (IQR) & $\begin{array}{c}3.05(2.40-3.90) \\
n=10\end{array}$ & $\begin{array}{c}3.80(3.30-4.50) \\
n=102\end{array}$ & $372.50(112)^{1}$ & .049 \\
\hline Mdn serum albumin (gm/dL) (IQR) & $\begin{array}{c}2.90(2.60-3.30) \\
n=11\end{array}$ & $\begin{array}{c}3.10(2.70-3.40) \\
n=123\end{array}$ & $655.50(134)^{1}$ & .48 \\
\hline Mdn serum bone-specific alk. phosphatase (mcg/L) (IQR) & $\begin{array}{c}10.20(8.95-17.40) \\
n=4\end{array}$ & $\begin{array}{c}8.85(6.40-12.90) \\
n=50\end{array}$ & $140.50(54)^{1}$ & .33 \\
\hline Mdn serum parathyroid hormone (pg/mL) (IQR) & $\begin{array}{c}47.00(39.00-53.00) \\
n=11\end{array}$ & $\begin{array}{c}59.50(33.50-88.50) \\
n=104\end{array}$ & $479.00(115)^{1}$ & .13 \\
\hline Mdn serum vitamin D 25-OH (ng/mL) (IQR) & $\begin{array}{c}22.00(21.00-33.00) \\
n=13\end{array}$ & $\begin{array}{c}25.00(17.00-35.00) \\
n=138\end{array}$ & $1,031.00(151)^{1}$ & .78 \\
\hline Mdn serum creatinine (mg/dL) (IQR) & $\begin{array}{c}1.00(0.90-1.10) \\
n=17\end{array}$ & $\begin{array}{c}0.94(0.80-1.20) \\
n=190\end{array}$ & $1641.50(207)^{1}$ & .59 \\
\hline
\end{tabular}

Note: Mdn: median, IQR: interquartile range, SD: standard deviation, and df: degrees of freedom for the test statistic (i.e., ${ }^{1}$ Wilcoxon rank sum test due to nonnormality or low sample size in the fracture cohort; ${ }^{2}$ Pearson's chi-square statistic; ${ }^{3}$ Fisher's exact test due to low expected frequencies; and ${ }^{4}$ independent $t$-test with observed normality and homogeneity). Percentages are within column. 
TABLE 2: Comparison of reasons for transplantation in those with fractures versus no fractures in the lung transplant population.

\begin{tabular}{lll}
\hline & Fracture $n=17$ & Nonfracture $n=193$ \\
\hline Reason for transplantation & & $60 / 193(31 \%)$ \\
Chronic obstructive pulmonary disease & $8 / 17(47 \%)$ & $64 / 193(33 \%)$ \\
Idiopathic pulmonary fibrosis & $5 / 17(29 \%)$ & $69 / 193(36 \%)$ \\
Other & $4 / 17(24 \%)$ & 25-Cystic fibrosis \\
& & 10-Sarcoidosis \\
& & 7-A1AD \\
& & 6-Scleroderma \\
& & 6-Bronchiolitis obliterans \\
& 3-Bronchiectasis \\
& 2-Sarcoidosis & 2-Lymphangioleiomyomatosis \\
& 1-A1AD & 2-Polymyositis \\
& 1-Scleroderma & 2-Pulmonary arterial hypertension \\
& & 1-Eosinophilic granuloma \\
& & 1-Desquamative interstitial pneumonia \\
& & 1-Hypereosinophilic syndrome \\
& & 1-Langerhan cell histiocytosis \\
& & 1-Usual interstitial pneumonia \\
\end{tabular}

fracture in heart transplant patients in years 1 to 5 was $3.8 \%, 1.4 \%, 1.9 \%, 0.5 \%$, and $0.5 \%$, respectively. The median time to the first fracture was 12 months and the mean time was $18.41 \pm 15.02$ months. Out of the 105 heart transplant recipients, 17 patients were female, and $11.8 \%$ of female patients incurred fractures. Out of the 88 male transplant recipients, $8.0 \%$ of male recipients incurred fractures.

The median pretransplant LS T score did not differ in those who fractured versus those who did not $(-0.80[-1.40-$ $0.30]$ versus $0.55[-0.90-1.60], P=.28)$. The median pretransplant femoral neck $\mathrm{T}$ score did not differ in those who fractured versus those who did not $(-1.30[-2.20--1.20]$ versus $-0.50[-1.30-0.20], P=.09)$.

The median posttransplant LS T score did not differ in those who fractured versus those who did not $(-0.50[-2.30-$ $0.00]$ versus $-0.20[-1.10-1.10], P=.21)$. The median femoral neck $\mathrm{T}$ score was statistically lower in the fracture group versus the nonfracture group $(-2.50[-3.10--2.30]$ versus $-1.00[-1.70--0.40], P=.003)$.

The median age (in years) of heart transplant recipients who fractured was not significantly different than those who did not (61.00 [55.00-68.00] versus 55.50 [46.50-62.00], $P=.051)$. The use of bisphosphonates in fracture and nonfracture groups did not differ in heart transplant patients (56\% versus $39 \%, P=.48$ ). The use of calcium and vitamin $\mathrm{D}$ in fracture and nonfracture groups did not differ in heart transplant patients (89\% versus $89 \%, P=.99)$. The use of glucocorticoids in fracture and nonfracture groups did not differ in heart transplant patients (56\% versus $70 \%, P=.46$ ).

Fracture locations varied, including 3 patients with vertebral (33.3\%), 2 with femur (22.2\%), and 1 with a foot fracture (11.1\%) alone. One patient had both vertebral and femur fractures (11.1\%), 1 patient had both hip and shoulder fractures (11.1\%), and 1 had humeral, tibial, and toe fractures (11.1\%) concurrently. None of these patients incurred subsequent fractures. Etiology of those who fractured after transplant included 5 patients with idiopathic cardiomyopathy (ICM), 3 with dilated cardiomyopathy (DCM), and 1 with viral cardiomyopathy (VCM). Out of the 4 patients who had fractures prior to heart transplantation, none had fractures following transplantation.

\section{Discussion}

The results of our descriptive study demonstrate that fragility fractures following lung transplantation and heart transplantation occur to a lesser degree compared with prior studies.

4.1. Lung Transplantation. There is a paucity of available studies assessing fracture risk following lung transplantation, mainly published in the late 1990 s and early 2000 s. In the first year after lung transplantation, symptomatic fracture rate of $15-50 \%$ and a loss of bone mineral density (BMD) of $4 \%$ to $12 \%$ have been reported [4]. Bone mineral density loss is greatest in the first 6 months following transplantation because immunosuppressive medications induce high bone turnover and uncoupling of bone formation and resorption. Patients who are evaluated for lung transplantation have a high prevalence of low BMD and osteoporosis, increasing the risk of fracture after transplantation [5]. A prospective study in 1999 demonstrated a high incidence of fractures in the first year after lung transplantation, despite receiving therapy with calcium, vitamin $\mathrm{D}$, or an agent to inhibit bone resorption within the first month. On average, there was a $1.3 \%$ loss in BMD at the lumbar spine and $2.8 \%$ loss in BMD at the femoral neck, but $50 \%$ of the patients sustained a significant decline in BMD at one or both of these sites [6]. Despite these low rates of bone loss, the fracture rate remained high, with $46 \%$ of women and $13 \%$ of men sustaining fractures. Another 
TABLE 3: Comparison of those with fractures versus no fractures in the heart transplant population.

\begin{tabular}{|c|c|c|c|c|}
\hline & Fracture $n=9$ & Nonfracture $n=96$ & Test statistic (df) & $P$ \\
\hline \multicolumn{5}{|l|}{ Demographics } \\
\hline Mdn age (years) (IQR) & $\begin{array}{c}61.00(55.00-68.00) \\
n=9\end{array}$ & $\begin{array}{c}55.50(46.50-62.00) \\
n=96\end{array}$ & $648.00(105)^{1}$ & .051 \\
\hline Mdn BMI (IQR) & $\begin{array}{c}23.55(22.57-28.31) \\
n=9\end{array}$ & $\begin{array}{c}24.62(21.98-28.94) \\
n=96\end{array}$ & $453.00(105)^{1}$ & .79 \\
\hline Females & $2 / 9(22 \%)$ & $15 / 96(16 \%)$ & $0.26(1)^{2}$ & .64 \\
\hline Menopause & $1 / 2(50 \%)$ & $5 / 15(33 \%)$ & $0.22(1)^{2}$ & .99 \\
\hline Ex-smokers & $5 / 9(56 \%)$ & $51 / 96(53 \%)$ & $0.02(1)^{2}$ & .99 \\
\hline Pretransplant fracture & 0 & $4 / 95(4 \%)$ & $0.39(1)^{2}$ & .99 \\
\hline \multicolumn{5}{|l|}{ Reason for transplantation } \\
\hline Ischemic cardiomyopathy (ICM) & $5 / 9(56 \%)$ & $38 / 96(40 \%)$ & & \\
\hline Dilated cardiomyopathy (DCM) & $3 / 9(33 \%)$ & $49 / 96(51 \%)$ & $1.35(2)^{2}$ & .51 \\
\hline Other & $1 / 9(11 \%)$ & $9 / 96(9 \%)$ & & \\
\hline \multicolumn{5}{|l|}{ Pretransplant values } \\
\hline Mdn LS T score (IQR) & $\begin{array}{c}-0.80(-1.40-0.30) \\
n=3\end{array}$ & $\begin{array}{c}0.55(-0.90-1.60) \\
n=42\end{array}$ & $44.00(45)^{1}$ & .28 \\
\hline Mdn femoral neck T score (IQR) & $\begin{array}{c}-1.30(-2.20--1.20) \\
n=3\end{array}$ & $\begin{array}{c}-0.50(-1.30-0.20) \\
n=45\end{array}$ & $33.50(48)^{1}$ & .09 \\
\hline Mdn serum calcium (mg/dL) (IQR) & $\begin{array}{c}8.90(8.20-9.10) \\
n=9\end{array}$ & $\begin{array}{c}8.90(8.40-9.20) \\
n=95\end{array}$ & $448.50(104)^{1}$ & .79 \\
\hline Mdn serum phosphorus (mg/dL) (IQR) & $\begin{array}{c}4.30(3.40-4.50) \\
n=9\end{array}$ & $\begin{array}{c}3.65(2.70-4.20) \\
n=92\end{array}$ & $553.00(101)^{1}$ & .26 \\
\hline Mean serum albumin (gm/dL) (SD) & $\begin{array}{c}3.10(2.40-3.20) \\
n=9\end{array}$ & $\begin{array}{c}3.00(2.50-3.50) \\
n=93\end{array}$ & $432.50(102)^{1}$ & .72 \\
\hline Mdn serum bone-specific alk. phosphatase (mcg/L) (IQR) & $n=0$ & $\begin{array}{c}8.75(6.50-11.0) \\
n=2\end{array}$ & - & - \\
\hline Mdn serum parathyroid hormone (pg/mL) (IQR) & $n=0$ & $\begin{array}{c}104.00(29.50-153.50) \\
n=13\end{array}$ & - & - \\
\hline Mdn serum vitamin D 25-OH (ng/mL) (IQR) & $\begin{array}{c}30.50(25.00-36.00) \\
n=2\end{array}$ & $\begin{array}{c}26.00(6.00-34.50) \\
n=9\end{array}$ & $13.50(11)^{1}$ & .73 \\
\hline Mdn serum creatinine (mg/dL) (IQR) & $\begin{array}{c}1.20(1.00-1.40) \\
n=9\end{array}$ & $\begin{array}{c}1.40(1.10-1.67) \\
n=95\end{array}$ & $381.50(104)^{1}$ & .30 \\
\hline \multicolumn{5}{|l|}{ Posttransplant medications } \\
\hline Bisphosphonate & $5 / 9(56 \%)$ & $37 / 96(39 \%)$ & $0.99(1)^{2}$ & .48 \\
\hline Calcium and vitamin $\mathrm{D}$ & $8 / 9(89 \%)$ & $84 / 94(89 \%)$ & $0.002(1)^{2}$ & .99 \\
\hline Glucocorticoid & $5 / 9(56 \%)$ & $67 / 96(70 \%)$ & $0.77(1)^{3}$ & .46 \\
\hline \multicolumn{5}{|l|}{ Posttransplant values } \\
\hline Mdn LS T score (IQR) & $\begin{array}{c}-0.50(-2.30-0.00) \\
n=7\end{array}$ & $\begin{array}{c}-0.20(-1.10-1.10) \\
n=58\end{array}$ & $171.50(65)^{1}$ & .21 \\
\hline Mdn femoral DXA T score (IQR) & $\begin{array}{c}-2.50(-3.10--2.30) \\
n=7\end{array}$ & $\begin{array}{c}-1.00(-1.70--0.40) \\
n=57\end{array}$ & $93.00(64)^{1}$ & .003 \\
\hline Mdn serum calcium (mg/dL) (IQR) & $\begin{array}{c}8.70(8.20-9.20) \\
n=9\end{array}$ & $\begin{array}{c}8.90(8.50-9.10) \\
n=94\end{array}$ & $397.50(103)^{1}$ & .41 \\
\hline Mdn serum phosphorus (mg/dL) (IQR) & $\begin{array}{c}3.60(3.40-3.80) \\
n=9\end{array}$ & $\begin{array}{c}3.60(3.10-4.40) \\
n=94\end{array}$ & $418.50(103)^{1}$ & .57 \\
\hline Mean serum albumin (gm/dL) (SD) & $\begin{array}{c}3.00(2.00-3.00) \\
n=9\end{array}$ & $\begin{array}{c}3.10(2.80-3.40) \\
n=92\end{array}$ & $289.50(101)^{1}$ & .04 \\
\hline Mdn serum bone-specific alk. phosphatase (mcg/L) (IQR) & $\begin{array}{c}16.60(10.30-22.30) \\
n=4\end{array}$ & $\begin{array}{c}13.70(9.20-15.50) \\
n=13\end{array}$ & $38.00(17)^{1}$ & .87 \\
\hline Mdn serum parathyroid hormone (pg/mL) (IQR) & $\begin{array}{c}68(5-78) \\
n=5\end{array}$ & $\begin{array}{c}100(48-133) \\
n=27\end{array}$ & $49.00(32)^{1}$ & .09 \\
\hline Mdn serum vitamin D 25-OH (ng/mL) (IQR) & $\begin{array}{c}25.50(24.50-29.50) \\
n=8\end{array}$ & $\begin{array}{c}19.00(14.50-28.50) \\
n=36\end{array}$ & $211.50(44)^{1}$ & .35 \\
\hline Mdn serum creatinine $(\mathrm{mg} / \mathrm{dL})(\mathrm{IQR})$ & $\begin{array}{c}1.50(1.00-1.70) \\
n=9\end{array}$ & $\begin{array}{c}1.40(1.00-1.83) \\
n=93\end{array}$ & $470.50(102)^{1}$ & .94 \\
\hline
\end{tabular}

Note: Mdn: median, IQR: interquartile range, SD: standard deviation, and df: degrees of freedom for the test statistic (i.e., ${ }^{1}$ Wilcoxon $W$ due to low sample size in the fracture cohort and ${ }^{2}$ Fisher's exact test due to low expected frequencies). Percentages are within column. 
TABLE 4: Comparison of reasons for transplantation in those with fractures versus no fractures in the heart transplant population.

\begin{tabular}{lll}
\hline & Fracture $n=17$ & Non-Fracture $n=193$ \\
\hline Reason for Transplantation & & $38 / 96(40 \%)$ \\
Ischemic Cardiomyopathy (ICM) & $5 / 9(56 \%)$ & $49 / 96(51 \%)$ \\
Dilated Cardiomyopathy (DCM) & $3 / 9(33 \%)$ & $9 / 96(9 \%)$ \\
Other & $1 / 9(11 \%)$ & $-2-$ Marfan's Syndrome \\
& & -2 -Familial DCM \\
& $-1:$ Viral Cardiomyopathy & -2 -Restrictive Cardiomyopathy \\
& & -2 -Viral Cardiomyopathy \\
& & -1 -Redo Right Ventricular Failure \\
\hline
\end{tabular}

study from 1996 demonstrated prevalent fractures in $11 \%$ of 55 patients awaiting lung transplantation and $27 \%$ of 45 lung transplant recipients [5]. In 2000, $18 \%$ (5 out of 28 ) of patients suffered osteoporotic fractures within 6 to 12 months after transplantation [7].

Earlier studies demonstrated high incidences of fragility fractures despite supplemental treatment to preserve bone mass. One study demonstrated a decrease in BMD at the lumbar spine and femoral neck despite prophylactic treatment with calcium and vitamin D [7]. Other studies demonstrated high incidences of fragility fractures despite therapy with calcium, vitamin $\mathrm{D}$, or antiresorptive medications $[6,7]$. One study demonstrated a $47.3 \%$ deficiency of $25-\mathrm{OHD}$ $(<30 \mathrm{ng} / \mathrm{mL})$, which was independently associated with a lower FEV1 and more severe B-grade rejections [8].

In our patient population, the majority of patients were treated with calcium and vitamin $\mathrm{D}$, which was not statistically significant between groups. Approximately half of the patients were treated with bisphosphonate therapy in both groups, which did not differ between both groups. In a pilot study, aggressive antiresorptive therapy preserved or increased BMD prior to and following transplantation by administration of a bisphosphonate prior to and following transplantation, resulting in only $4 \%$ of symptomatic fractures, compared to our current study fracture rate of $8 \%$ [9]. The status of antiresorptive therapy prior to transplantation in our study is not known.

Similar to previous studies, a reduction in BMD was present in patients prior to and following transplantation $[3,5]$. Patients in both the fracture and nonfracture groups were in the osteopenia range for BMD and did not differ significantly prior to transplantation. Patients who fractured had a statistically significant difference in femoral neck BMD following transplantation but not the lumbar spine. Consistent with multiple studies, despite antiresorptive therapy and calcium/vitamin D, bone loss persisted.

4.2. Cardiac Transplantation. Fragility fractures are prevalent in the heart transplant population. Studies have demonstrated an incidence of vertebral fractures ranging from 1 to $44 \%$ and osteonecrosis of the femoral head in 1-9\% of heart transplant recipients [2]. In a cross-sectional study evaluating 40 patients two years after cardiac transplantation, severe osteoporosis was seen at the lumbar spine in $28 \%$ and at the femoral neck in $20 \%$ of the patients. In addition, $35 \%$ of the patients had one or more vertebral fractures [10].

Rapid bone loss occurred within the first year following heart transplantation [10]. In patients who received vitamin $\mathrm{D}$ and calcium supplementation, without an antiresorptive agent, the incidence of bone loss at the lumbar spine based on mean percent BMD change was $7.3 \%$ and at the femoral neck was $10.5 \%$ during the first year after cardiac transplantation, with $92 \%$ of the patients suffering a significant bone loss during this year [1].

In heart transplant patients, the incidence of fracture was highest in the first year following transplantation. It has been observed in other studies that, after the initial year, there is usually a marked decline in the cumulative glucocorticoid dose and a decrease in immunosuppressive therapy [11]. The use of steroid pulses for rejection is less likely after one year; maintenance steroids are at a lower dose, and there is an annual increase in trabecular bone that persists for a minimum of 3 years.

In our patient population, there was a statistically significant decline in femoral neck BMD following transplantation. This was not seen in the lumbar spine prior to or following transplantation. These findings were similar to a prior study in which the BMD in the femur was below in both cardiac transplant candidates and recipients [2]. Prior to transplant, the use of loop diuretics may stimulate a secondary increase in PTH causing a loss of bone density at the hip.

4.3. Transplantation Medications. Following transplantation, patients are placed on a drug regimen that commonly includes glucocorticoids and calcineurin inhibitors. Glucocorticoid use is a common cause of secondary osteoporosis in transplant patients, and calcineurin inhibitors have adverse effects on skeletal integrity. Both of these medications can lead to bone disease characterized by rapid bone loss and high rates of fractures [12]. The use of glucocorticoids is associated with an increased risk for the development of osteoporosis and fractures by increasing apoptosis of osteoblasts and impairing osteogenesis. It also causes an increase in osteoclastogenesis and bone resorption [13]. A large decrease in BMD occurs within the first 3-6 months due to the large doses of glucocorticoids used immediately after grafting [14]. In addition, the use of other antirejection medications such as cyclosporine and tacrolimus has been shown to cause 
increased osteoclastic bone resorption and osteoblastic bone formation $[12,15]$. The combination of calcineurin inhibitors with glucocorticoids can have severe consequences on bone density. Even with the use of multiple immunosuppressive medications to help decrease the dosage of glucocorticoids, the occurrence of osteoporosis and fractures persists.

Numerous studies are present which illustrate the decline in bone mineral density and the incidence of osteoporosis following solid organ transplantation despite the use of calcium, vitamin $\mathrm{D}$, and antiresorptive therapy. One study through Northwestern University assessed the occurrence of fractures in solid organ transplant recipients (kidney, liver, and pancreas). This demonstrated a high frequency in transplant recipients, as $27.4 \%$ of patients developed fractures 2-6 years following transplantation, although patients received less than 6 months of glucocorticoids as part of the immunosuppressive regimen [16].

There are several limitations to our analysis. The data was obtained through review of medical records. Outside DXAs were not captured in some individuals. The number of DXA scans available for review prior to transplantation was often less than that following transplantation. The observed number of fractures may be underestimated as the data regarding fracture incidence was obtained through review of imaging and self-reporting during office visits. Hormonal status was not assessed, including testosterone deficiency or the use of hormone replacement therapy.

Given the long waiting time between acceptance to the waiting list and transplantation, transplant candidates with osteoporosis should be treated aggressively so that bone health is optimized following transplantation. As glucocorticoids are a risk factor for fractures, it is important to consider treatment in patients such as bisphosphonates with low bone mass or osteopenia [6]. However, the optimal duration of antiresorptive therapy is not known [9]. It is also important to aggressively screen for vitamin D deficiency as a study in the lung transplant population demonstrated that vitamin D deficiency has been associated with higher rejection rates and infections [17]. Assessment of mobility and the use of physical therapy following transplantation are another consideration for the future.

\section{Conclusion}

Our findings demonstrate a much lower incidence of fractures following transplantation in heart and lung transplant recipients in comparison to earlier reports. Comprehensive bone care, including DXA scans, screening for vitamin D deficiency, and early initiation of antiresorptive therapy at the pretransplant or immediate posttransplant period, is possible contributors to these improved outcomes.

\author{
Abbreviations \\ DXA: Dual energy X-ray absorptiometry \\ LS: Lumbar spine \\ PTH: $\quad$ Parathyroid hormone \\ 25-OHD: 25-Hydroxyvitamin D \\ COPD: Chronic obstructive pulmonary disease
}

IPF: Idiopathic pulmonary fibrosis

CF: Cystic fibrosis

A1AD: Alpha 1 antitrypsin deficiency

BO: Bronchiolitis obliterans

LAM: Lymphangioleiomyomatosis

PAH: Pulmonary arterial hypertension

DCM: Dilated cardiomyopathy

ICM: Ischemic cardiomyopathy

VCM: Viral cardiomyopathy

RCM: Restrictive cardiomyopathy

RV: Right ventricular

SD: Standard deviation

IQR: Interquartile range

Mdn: Median

df: $\quad$ Degrees of freedom.

\section{Conflict of Interests}

The authors declare that there is no conflict of interests regarding the publication of this paper.

\section{Authors' Contribution}

Aileen Hariman participated in research design, participated in writing the paper, participated in performance of the research, and participated in data analysis. Charles Alex participated in research design and participated in writing the paper. Alain Heroux participated in research design. Pauline Camacho participated in research design and participated in writing the paper.

\section{Acknowledgments}

This research was supported by faculty and staff from the Clinical Research Office (CRO) at Loyola University Chicago, Health Sciences Division, 2160 S. First Ave., Fahey Building, Room 001, Maywood.

\section{References}

[1] E. Shane, M. Rivas, D. J. McMahon et al., "Bone loss and turnover after cardiac transplantation," Journal of Clinical Endocrinology and Metabolism, vol. 82, no. 5, pp. 1497-1506, 1997.

[2] A. H. Lee, R. L. Mull, G. F. Keenan et al., "Osteoporosis and bone morbidity in cardiac transplant recipients," American Journal of Medicine, vol. 96, no. 1, pp. 35-41, 1994.

[3] E. Shane, S. J. Silverberg, D. Donovan et al., "Osteoporosis in lung transplantation candidates with end-stage pulmonary disease," American Journal of Medicine, vol. 101, no. 3, pp. 262269, 1996.

[4] S. L. Ferrari, L. P. Nicod, J. Hamacher et al., "Osteoporosis in patients undergoing lung transplantation," European Respiratory Journal, vol. 9, no. 11, pp. 2378-2382, 1996.

[5] R. M. Aris, I. P. Neuringer, M. A. Weiner, T. M. Egan, and D. Ontjes, "Severe osteoporosis before and after lung transplantation," Chest, vol. 109, no. 5, pp. 1176-1183, 1996. 
[6] E. Shane, A. Papadopoulos, R. B. Staron et al., "Bone loss and fracture after lung transplantation," Transplantation, vol. 68, no. 2, pp. 220-227, 1999.

[7] A. Spira, C. Gutierrez, C. Chaparro, M. A. Hutcheon, and C. K. N. Chan, "Osteoporosis and lung transplantation: a prospective study," Chest, vol. 117, no. 2, pp. 476-481, 2000.

[8] S. E. Verleden, R. Vos, R. Geenens et al., "Vitamin D deficiency in lung transplant patients: is it important?" Transplantation, vol. 93, no. 2, pp. 224-229, 2012.

[9] B. C. Cahill, M. K. O’Rourke, S. Parker, J. C. Stringham, S. V. Karwande, and T. P. Knecht, "Prevention of bone loss and fracture after lung transplantation," Transplantation, vol. 72, no. 7, pp. 1251-1255, 2001.

[10] E. Shane, M. Rivas, S. J. Silverberg, T. S. Kim, R. B. Staron, and J. P. Bilezikian, "Osteoporosis after cardiac transplantation," American Journal of Medicine, vol. 94, no. 3, pp. 257-264, 1993.

[11] H.-U. Stempfle, C. Werner, S. Echtler et al., "Prevention of osteoporosis after cardiac transplantation: a prospective, longitudinal, randomized, double-blind trial with calcitriol," Transplantation, vol. 68, no. 4, pp. 523-530, 1999.

[12] A. Cohen and E. Shane, "Osteoporosis after solid organ and bone marrow transplantation," Osteoporosis International, vol. 14, no. 8, pp. 617-630, 2003.

[13] L. E. Nikkel, C. S. Hollenbeak, E. J. Fox, T. Uemura, and N. Ghahramani, "Risk of fractures after renal transplantation in the United States," Transplantation, vol. 87, no. 12, pp. 1846-1851, 2009.

[14] M. Aringer, H. P. Kiener, M. D. Koeller et al., "High turnover bone disease following lung transplantation," Bone, vol. 23, no. 5, pp. 485-488, 1998.

[15] S. Epstein, "Post-transplantation bone disease: the role of immunosuppressive agents and the skeleton," Journal of Bone and Mineral Research, vol. 11, no. 1, pp. 1-7, 1996.

[16] B. J. Edwards, A. Desai, J. Tsai et al., "Elevated incidence of fractures in solid-organ transplant recipients on glucocorticoidsparing immunosuppressive regimens," Journal of Osteoporosis, vol. 2011, Article ID 59179, 8 pages, 2011.

[17] E. M. Lowery, B. Bemiss, T. Cascino et al., "Low vitamin D levels are associated with increased rejection and infections after lung transplantation," Journal of Heart and Lung Transplantation, vol. 31, no. 7, pp. 700-707, 2012. 


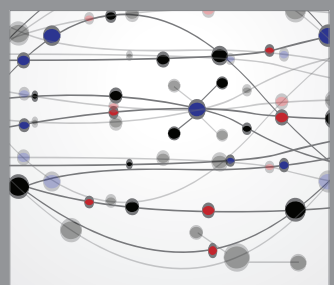

The Scientific World Journal
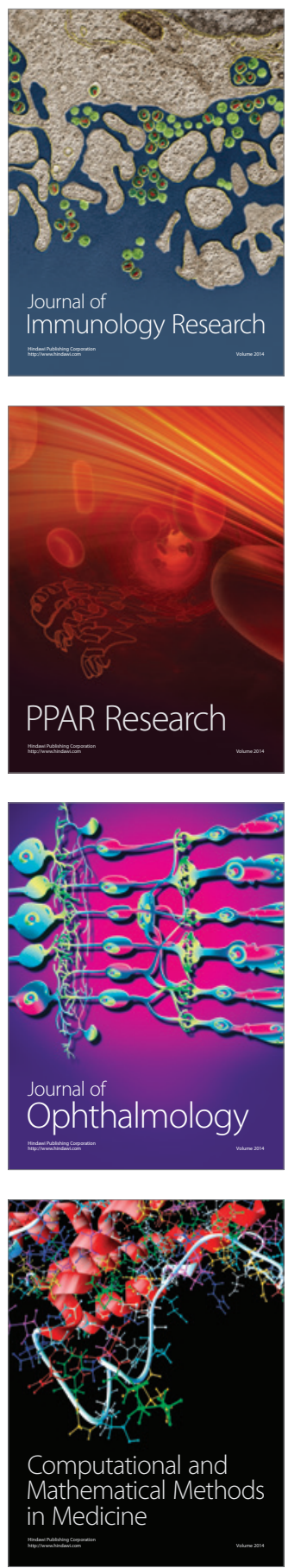

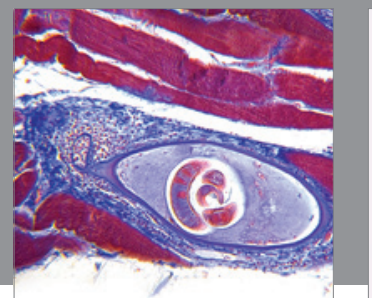

Gastroenterology

Research and Practice
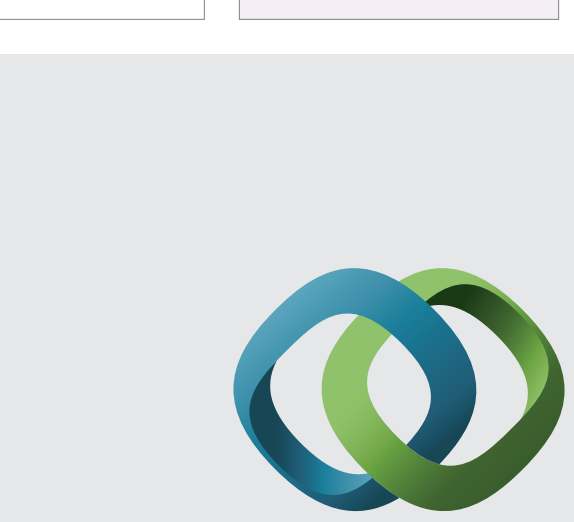

\section{Hindawi}

Submit your manuscripts at

http://www.hindawi.com
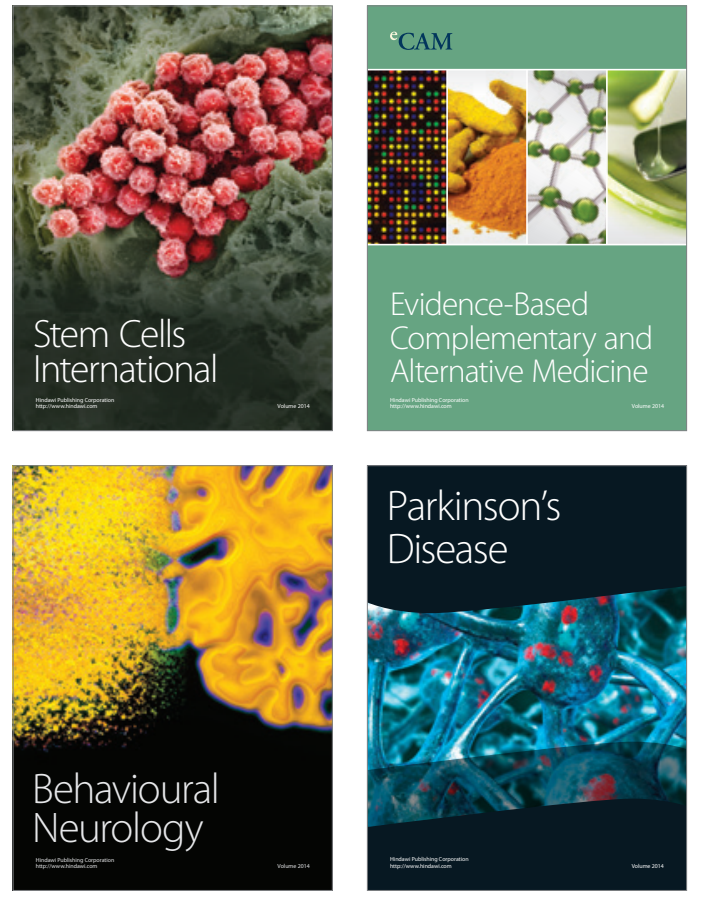
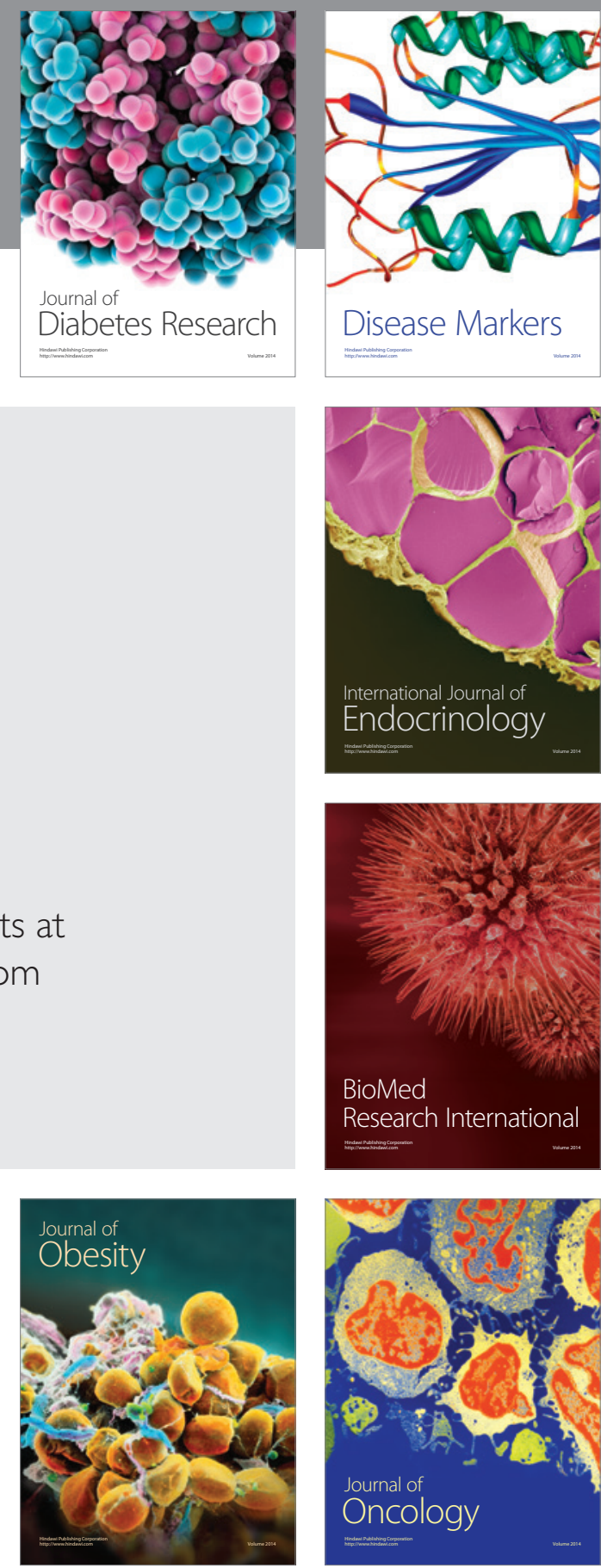

Disease Markers
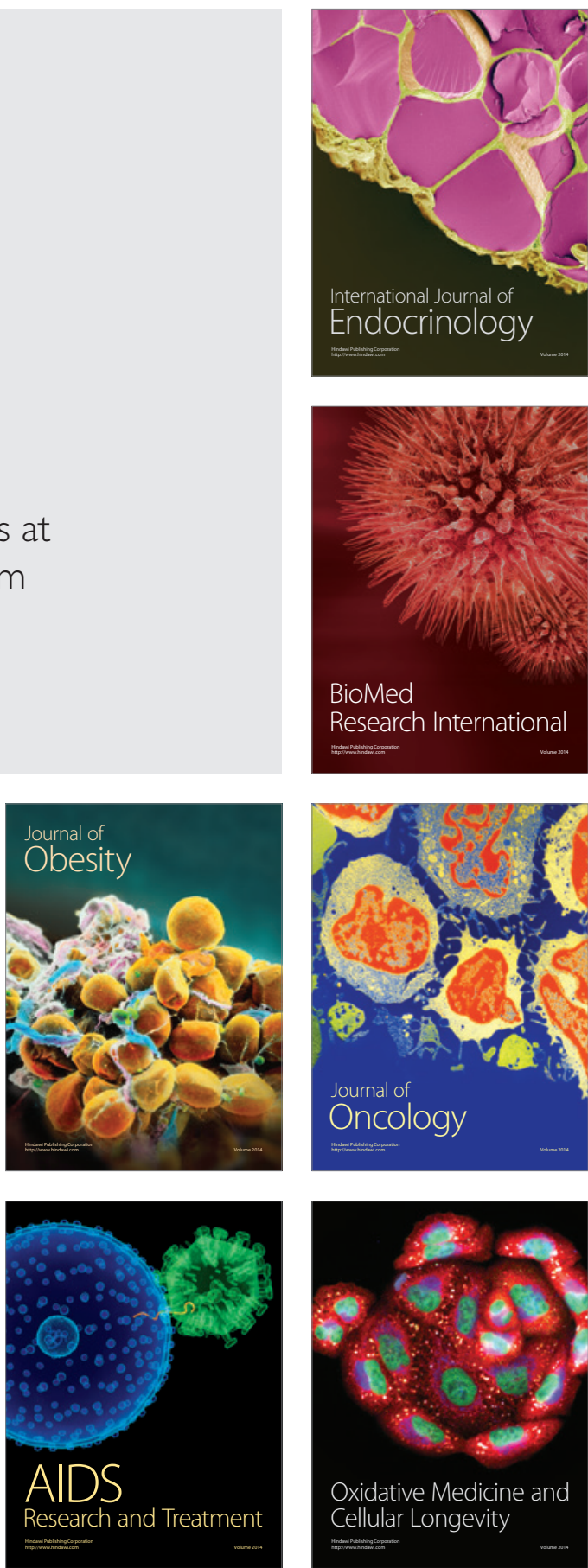